\title{
The Design and Calibration of Instrumented Particles for Assessing Water Infrastructure Hazards
}

\author{
Khaldoon Al-Obaidi, Yi Xu and Manousos Valyrakis * \\ Water Engineering Lab, School of Engineering, University of Glasgow, Glasgow G12 8QQ, UK; \\ 2372435A@student.gla.ac.uk (K.A.-O.); 2421380X@student.gla.ac.uk (Y.X.) \\ * Correspondence: Manousos.Valyrakis@glasgow.ac.uk; Tel.: +44-(0)-141-330-5209
}

Received: 9 May 2020; Accepted: 27 July 2020; Published: 30 July 2020

check for updates

\begin{abstract}
The highly dynamical entrainment and transport processes of solids due to geophysical flows is a major challenge studied by water infrastructure engineers and geoscientists alike. A miniaturised instrumented particle that can provide a direct, non-intrusive, low-cost and accessible method compared to traditional approaches for the assessment of coarse sediment particle entrainment is developed, calibrated and tested. The instrumented particle presented here is fitted with inertial microelectromechanical sensors (MEMSs), such as a triaxial accelerometer, a magnetometer and angular displacement sensors, which enable the recording of the particle's three-dimensional displacement. The sensor logs nine-axis data at a configurable rate of $200-1000 \mathrm{~Hz}$ and has a standard mode of deployment time of at least one hour. The data can be obtained and safely stored in an internal memory unit and are downloadable to a PC in an accessible manner and in a usable human-readable state. A plethora of improved design specifications have been implemented herein, including increased frequency, range and resolution of acceleration and gyroscopic sensing. Improvements in terms of power consumption, in comparison to previous designs, ensure longer periods of data logging. The embedded sensors are calibrated using simple physical motions to validate their operation. The uncertainties in the experiments and the sensors' readings are quantified and an appropriate filter is used for inertial sensor fusion and noise reduction. The instrumented particle is tested under well-controlled lab conditions, where the beginning of the destabilisation of a bed surface in an open channel flow, is showcased. This is demonstrative of the potential that specifically designed and appropriately calibrated instrumented particles have in assessing the initiation and occurrence of water infrastructure hazards.
\end{abstract}

Keywords: inertial sensor fusion; instrumented particle; MEMS; sediment entrainment; sensor calibration; frequency of entrainment

\section{Introduction}

The surface of a planet is shaped by geomorphic processes, the majority of which are driven by a range of geophysical flows, including turbulent fluvial, aeolian, snow/ice and lava flows. Of special interest for geomorphologists and civil and environmental engineers alike are turbulent flows in rivers, canals, estuaries and coasts that can set into entrainment and transport coarse sediment particles, which have the potential to destabilise critical infrastructure found on their way and result in significant geophysical hazards. Coarse particle entrainment in turbulent flows has been shown to occur due to the existence of strong energetic events that can result in its removal from its resting location [1]. Such transport processes are considered to be the governing mechanism for the failure of built infrastructure, such as bridge piers and abutments, as well as the destabilisation of riverbanks and embankments, rendering imperative the monitoring of the starting phase of these processes to reduce risk and improve resilience [2-4]. These processes depend on the particle and flow parameters, 
in addition to any specific water infrastructure designs present at the studied area. Despite recent advances in the prediction of the turbulent flow conditions that may result in entraining a particle [5-7], such criteria have yet to find widespread practical application in engineering. Instead, relatively expensive, indirect and highly inaccurate methods for detecting mean flow parameters to determine the possibility of sediment entrainment are still employed. In general, such studies use expensive acoustic Doppler velocimetry [8], laser Doppler velocimetry [9] or water level stations, along with discharge hydrographs, requiring regular or reactive visits to remote and harsh sites [10]. Recent technological advancements make it possible for sediment entrainment to be assessed directly, using novel tools, instead of traditionally monitoring surrogate flow metrics [2,11]. Using sensors in the field of riverbed sediment transport has been explored by multiple researchers [12-19]. However, none of the work presented in literature has used such smart sensors to establish a link between logged readings and sediment entrainment based on derived performance indicators. This work introduces the methodology to derive performance indicators, like frequency of entrainment, based on the particle's fused data, which can give information on the risk of earth surface destabilisation or the risk of scour development. Additionally, the presented instrumented particle in this work has a plethora of improved design specifications compared to other tools that exist in the literature, including a decreased particle, increased frequency and range of sensor readings, reduced error after calibration and fusion and improved battery life for longer deployment periods.

In this study, a miniaturised instrumented particle has been designed and developed to help monitor the above entrainment processes. The presented design capitalises on improvements in hardware, software and casing designs, stemming from the many years' experience in further developing a number of earlier prototypes [1-3], to allow improved and higher resolution particle motion detection. Additionally, this work presents the calibration and testing of the instrumented particle entrainments due to the action of near bed turbulent flow events at one of the open channel flumes of the Water Engineering Lab at the University of Glasgow, and the inertial sensor fusion of logged readings to achieve uncertainty reduction in the gathered dynamical data. Finally, the research herein suggests using MEMS instrumented particles laying on the riverbed surface for monitoring its destabilisation potential, using the frequency of entrainment as a performance indicator (Supplementary Materials).

To achieve this task, a $40 \mathrm{~mm}$ diameter data-logging device was designed, encapsulated in a 3D-printed plastic spherical shell. The device was designed for use in a laboratory setting (such as in water and sediment recirculating flumes), as well for as real world testing, on the bed surface of streams and rivers, for monitoring the potential for geomorphic work. Initial designs of the instrumented particle ranged from 125 to $75 \mathrm{~mm}$ in size [1-3]. However, riverbeds are characterised by a wider range of bed surface material and, in many cases, the characteristic size of sediment particles may be smaller than that of the previous designs. The decrease in size of the proposed instrumented particle was necessary in order to allow for widening its range of application by enabling the matching of the particle characteristics and assessing the possibility of sediment entrainment for certain flow conditions more effectively [1-3].

In addition to the reduced size, additional improvements in design specifications included a higher frequency and range of acceleration and gyroscopic sensing, and inertial sensor fusion for reducing the uncertainty in tracking the motion of the particle. Hardware modifications, resulting in lower power consumption, allowed for achieving longer logging periods. The modular design can be extended to embed a range of additional sensors, such as magnetometers, water temperature sensors, static and dynamic pressure transducers (for flow depth monitoring and dynamic flow motions) and photocell sensors (for ecological applications). Even though those sensors have a promising potential for future deployment in a wider range of ecohydraulic and geoscience applications, focus was given to the fusion of triaxial acceleration and gyroscope readings with the magnetometer.

The data rate of the sensors ranged from $200 \mathrm{~Hz}$ up to $1000 \mathrm{~Hz}$. A one-hour run time was also required for robust field deployment and recording the instrumented particle's inertial data. Reducing the size of the instrumented particle was a challenging task, as it required a high level of integration 
and optimisation of the internal components, both in terms of physical space (circuit design) and power consumption. The physical restrictions imply that function was improved, while the instrumented particle's volume was reduced by more than nine times compared to previous designs, meaning that all subsystems were integrated completely into a truly bespoke solution, as discussed in the following sections.

The rest of this paper consists of five sections that present: the design considerations, the calibration, the flume testing, the results and discussion and the conclusions and future work. Section 2 introduces the main considerations for the design of the instrumented particle for the applications discussed previously. Section 3 describes the calibration process to estimate the uncertainties in the sensor's readings and the inertial sensor fusion filter used to reduce these uncertainties. Section 4 presents the flume testing of the instrumented particle by performing three experiments for a certain flowrate range that represents the near-threshold conditions. Section 5 introduces the results and demonstrates how the sensor's logged readings could be used to assess the probability of sediment entrainment. Finally, Section 5 presents the conclusions and future work.

\section{Design Considerations}

The main considerations for the design of the instrumented particle are: the size and availability of the sensor, the energy supply, the flash storage, the microcontroller, the inertial sensor, weight balance and data transfer [20]. The target design requirements for the instrumented particle and the sensor are described in Table 1 below:

Table 1. Main design considerations.

\begin{tabular}{cc}
\hline Size of instrumented particle & $\leq 4 \mathrm{~cm}$ in diameter \\
\hline Size of sensor & $\leq 4 \mathrm{~cm}$ in diameter \\
\hline Time of operation & $>1 \mathrm{~h}$ \\
\hline Logging frequency range & $>200 \mathrm{~Hz}$ \\
\hline Maximum acceleration & $>10 \mathrm{~g}$ \\
\hline Maximum rotational velocity & As fast and practical as possible \\
\hline Data transfer rate from sensor to PC & Cost effective and practical \\
\hline Energy supply & Predictable access, erase and write times. Logging speed of $>5 \mathrm{~KB} / \mathrm{s}$ \\
\hline Flash storage & Simplicity, strong documentation and availability of test data \\
\hline Microcontroller & Six or more axes (triaxial accelerometer and triaxial gyroscope) \\
\hline Inertial sensor & Communication over I2C or SPI \\
\hline
\end{tabular}

Based on the criteria specified in Table 1, the final decision is made to select Invensense MPU-9250 inertial sensor (Invensense Inc., San Jose, CA, USA). It supports three-axis acceleration, three-axis rotational velocity, and a three-axis magnetometer, as well as a well-renowned internal Digital Motion Processor, supporting up to $16 \mathrm{~g}$ of measurable acceleration, up to $2000^{\circ} \mathrm{s}$ of measurable rotational velocity and sensor output frequency well in excess of $200 \mathrm{~Hz}$ [21]. As for the energy supply, rechargeable coin cells (Varta Microbattery produced under CoinPower [22], Varta Microbattery GmbH, Ellwangen Germany) are selected due to being a cost effective and practical option compared to lithium batteries [23,24]. For the flash storage, discrete flash integrated circuits are chosen due to more predictable access, erase and write times compared to an SD card. S25FL128S (Cypress Semiconductor Corporation, San Jose, CA, USA) is chosen specifically due its low cost, its $16 \mathrm{MB}$ size and high data logging speed of $1.5 \mathrm{MB} / \mathrm{s}$, which is far faster than the required $5 \mathrm{~KB} / \mathrm{s}$ (transferring roughly $16 \mathrm{MB}$ of logged data for one hour of field deployment). As for the microcontroller, the Arduino Zero (Adruino, Ivrea Italy) is chosen for its higher likelihood of communicating, computing and storing data within the necessary time frame, 
as shown in Figure 1. The final design of the sensor is shown in Figure 2 and specific attention has been paid to centring the weight of the sensor to produce a well-balanced instrumented particle. Finally, for data transfer, Tera Term, an open source software, is used to establish the communication between the microcontroller and the inertial sensor. The software allows the user to erase data from the flash, transfer data to the SD card and start the logging process. The rate of data transfer from the flash to the SD card (even though it can be further optimised) is sufficiently fast (requiring about half of the deployment time to transfer the logged readings).

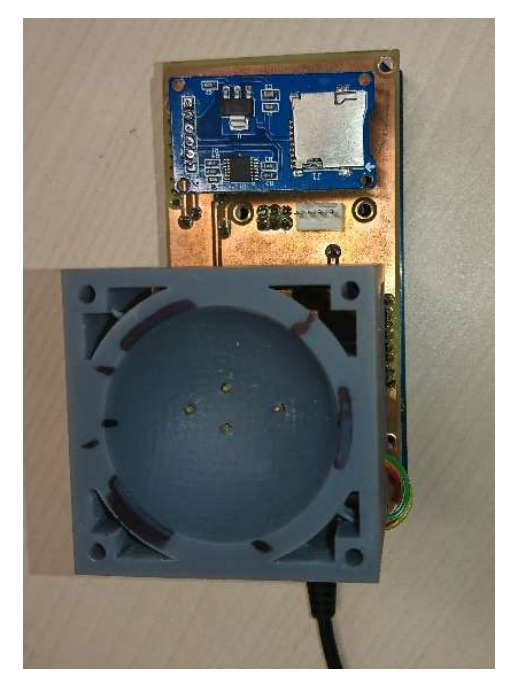

Figure 1. The microcontroller and docking station used for charging the inertial sensor and transferring the data to an SD card.

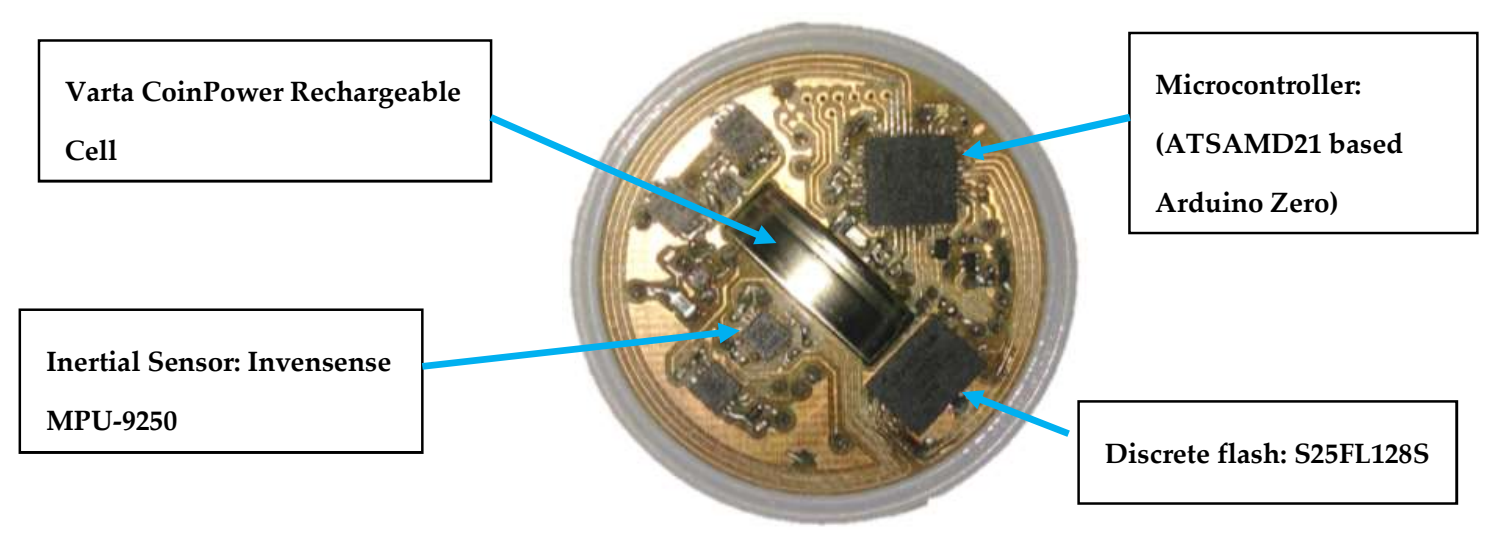

Figure 2. The $40 \mathrm{~mm}$ instrumented particle printed circuit board design.

\section{Calibration and Uncertainty Estimation}

\subsection{Checking Sensor Response and Range for Vigorous Rolling Motion}

Before the calibration process starts, a quick sensing check and endurance experiment is performed. This involves inserting the sensors into the spherical shell of the instrumented particle $(4 \mathrm{~cm}$ in external diameter), fixing them properly so that they are centred at their centre of gravity and allowing them to vigorously roll on a flat surface, by hand. The resulting motion is checked by separately observing the readings of the accelerometer, gyroscope and magnetometer over the three axes, as shown in Figure 3. 

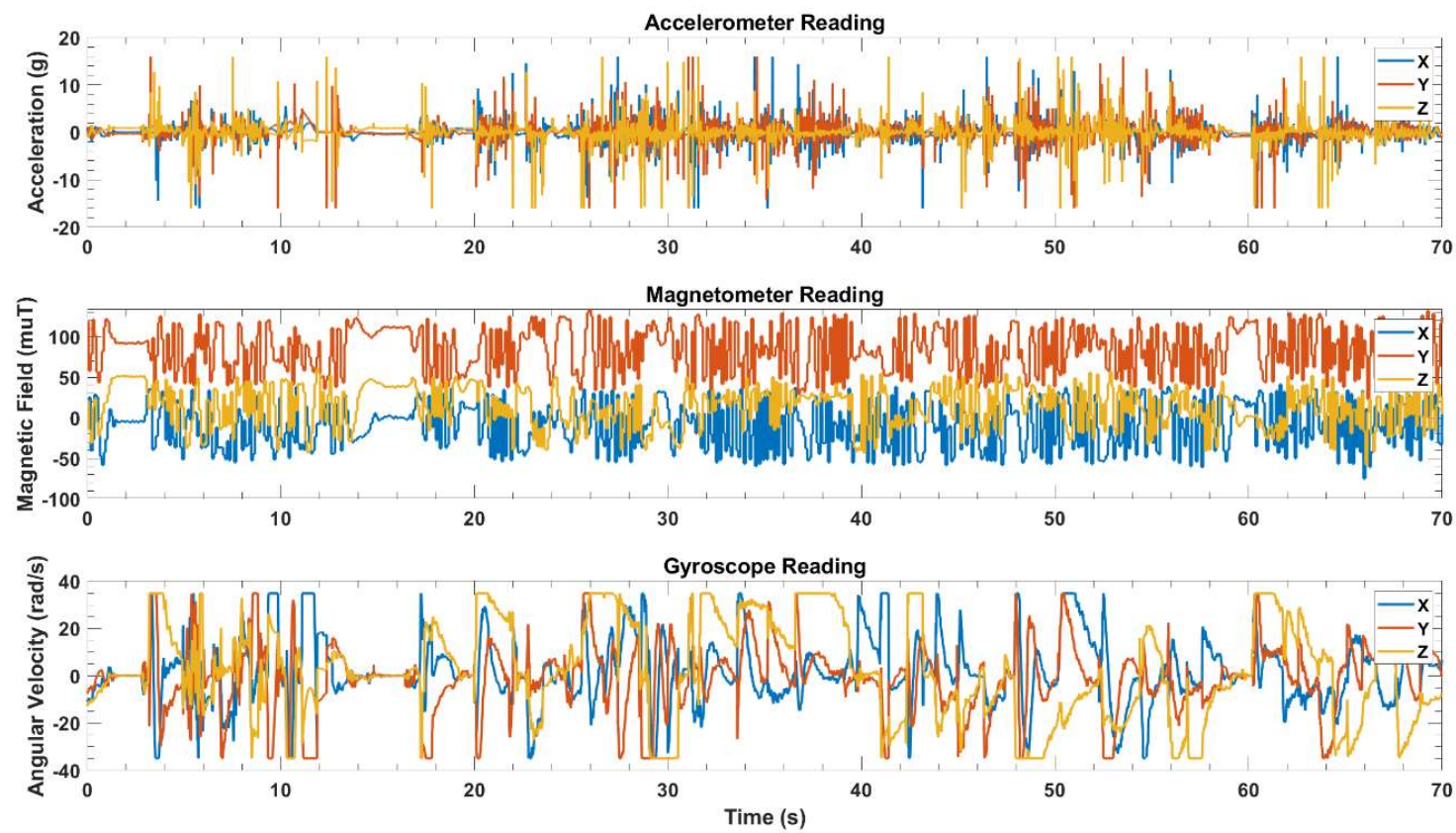

Figure 3. Accelerometer, gyroscope and magnetometer logged readings during a quick sensing check and endurance experiment that is performed by throwing the sensor to roll on the floor.

From this simple test, it is observed that the selected ranges of $16 \mathrm{~g}$ and $2000 \% \mathrm{~s}$ are sufficient for recording the maximum particle accelerations and gyroscope readings. Additionally, the accelerometer recorded the gravitational acceleration (shown as a recording of $1 \mathrm{~g}$ ) on the $z$-axis during the times when the sensor is static. Finally, after throwing the sensor with a high force, it remains operating and functional, which confirms that it could be used in harsh and remote water environments, such as in fluvial systems.

\subsection{Calibration for Controlled Harmonic Motion}

The calibration process consisted of individually calibrating the accelerometer and the gyroscope. Specifically, for the calibration of the accelerometer, a simple harmonic motion of a pendulum with the instrumented particle at its centre of gravity and a shaking table of a known acceleration range are used. The pendulum test consists of two parts, where the first part involves measuring the pendulum angle and checking the accelerometer's reading at the lowest point of the swing, and the second involves the oscillation period of the pendulum, using a stop watch and the accelerometer's readings and comparing both to the oscillation period that is estimated theoretically. As for the first step, the accelerometer data are used to determine the total acceleration of the sensor using Equation (1). The total acceleration at the beginning of the experiment is zero since the sensor is let from rest then it increases in value till it reaches a maximum at the lowest point of the motion, since the angle is the highest, as illustrated in Figure 4. The acceleration of the sensor at the lowest point is then calculated using Equation (2).

$$
\mathrm{a}=\sqrt{\mathrm{a}_{\mathrm{x}}^{2}+\mathrm{a}_{\mathrm{y}}^{2}+\mathrm{a}_{\mathrm{z}}^{2}}
$$

where $\mathrm{a}_{\mathrm{x}}, \mathrm{a}_{\mathrm{y}}$ and $\mathrm{a}_{\mathrm{z}}$ are the $\mathrm{x}, \mathrm{y}$ and $\mathrm{z}$ components of the acceleration and

$$
\text { a@lowest point }=\mathrm{g} \sin \theta
$$

where $\theta$ is the angle of the pendulum, as illustrated in Figure 4, and $g$ is the gravitational acceleration $\left(9.81 \mathrm{~m} / \mathrm{s}^{2}\right)$. 


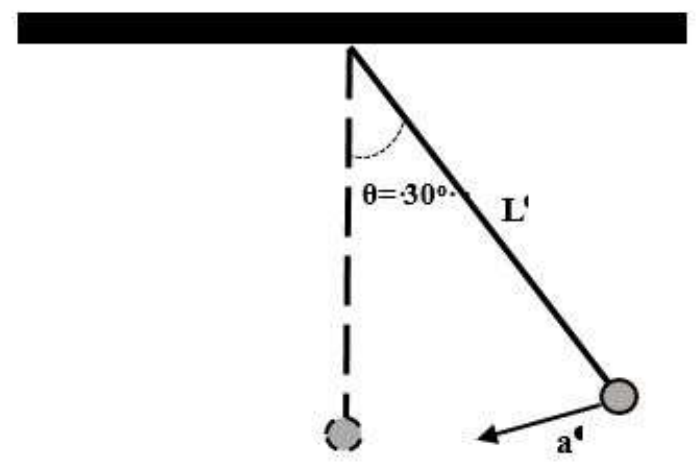

Figure 4. An illustration of a simple harmonic motion of a pendulum.

A starting pendulum angle $(\theta)$ of $30^{\circ}$ is selected, as shown in Figure 4. The experiment is repeated 10 times and the total acceleration results at the lowest point are estimated using (1), based on the accelerometer's readings for the 10 runs, as shown in Table 2. The standard deviation for the 10 runs is 0.0073 which is a small value, indicating a small spread in the results. The standard deviation per mean is estimated to be 0.0146 , indicating that the error in the experiment is small. As for the average error of the 10 runs, it is $1.08 \%$, which incorporates both experimental measurement errors (as the particle is released by hand) and estimation errors due to the mechanical energy lost due to friction with the air, not accounted for in Equation (2). Thus, the average error in the accelerometer's readings for this experiment is deemed to be acceptable.

Table 2. The acceleration results for 10 runs of pendulum swinging, starting from a known angle.

\begin{tabular}{cccc}
\hline Run Name & $\begin{array}{c}\text { Highest Recorded Total } \\
\text { Acceleration }\left(\mathbf{9 . 8 1} \times \mathbf{~ m} / \mathbf{s}^{\mathbf{2}}\right)\end{array}$ & $\begin{array}{c}\text { Expected Acceleration }\left(\mathbf{9 . 8 1} \times \mathbf{~ m} / \mathbf{s}^{\mathbf{2}}\right) \\
\text { Estimated Using Equation } \mathbf{( 2 )}\end{array}$ & \% Error \\
\hline Run1 & 0.516 & & 3.28 \\
Run2 & 0.492 & & 1.54 \\
Run3 & 0.508 & 0.500 & 1.6 \\
Run4 & 0.498 & 0.34 \\
Run5 & 0.497 & 0.56 \\
Run6 & 0.502 & 0.30 \\
Run7 & 0.503 & 0.54 \\
Run8 & 0.501 & 0.22 \\
Run9 & 0.489 & 2.2 \\
Run10 & 0.499 & 0.18 \\
\hline
\end{tabular}

The second part of the experiment is to estimate the duration of a full swing for the pendulum using the accelerometer's readings. The pendulum's single full swing period recorded by the accelerometer (Figure 5) corresponds to half a period of the pendulum motion [25]. The theoretical full swing period of a simple pendulum (the time it takes to swing back-and-forth) in this experiment, estimated using Equation (3), is $1.30 \mathrm{~s}$ :

$$
\mathrm{T}=2 \pi \sqrt{\frac{\mathrm{L}}{\mathrm{g}}}
$$

where $\mathrm{T}$ is the period of a simple pendulum, $\mathrm{L}$ is the length from the pivot point to the center of mass of the sensor ( $42 \mathrm{~cm}$ in this experiment) $\mathrm{g}$ is the gravitational acceleration. The experiment is repeated 10 times and the swing period results, estimated using the accelerometer's readings for the 10 runs, are shown in Table 3.

The standard deviation for the 10 periods of the 10 runs in the experiment is 0.0234 , which is a small value, indicating a small spread in the results. The standard deviation per mean is estimated to be 0.0180 , indicating that the error in the experiment is small. The average error in the 10 runs is $1.41 \%$ 
and, considering the uncertainties in assessing the parameters in Equation (3) and the error due to the mechanical energy lost due to friction with the air, it is deemed to be acceptable.

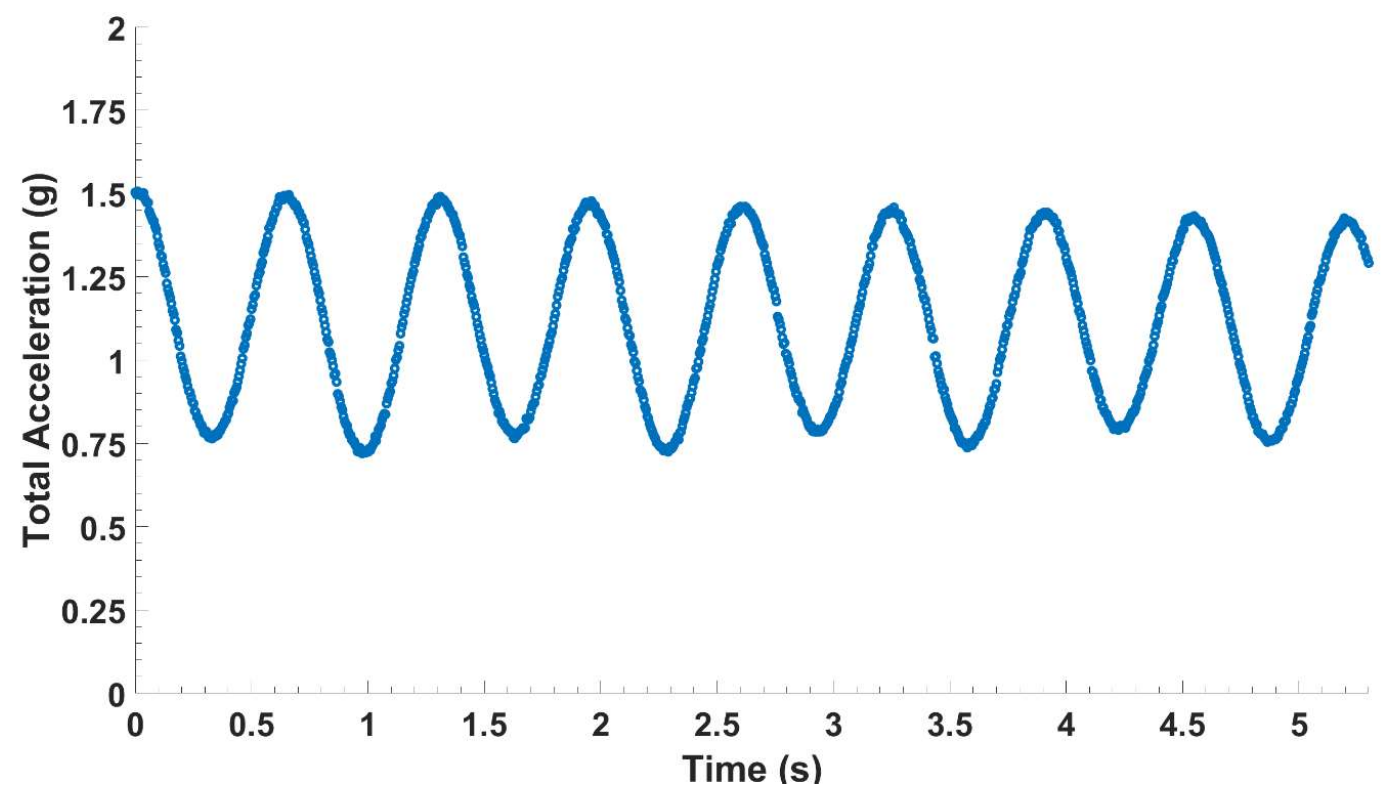

Figure 5. Total acceleration estimated using the accelerometer logged readings corresponds to half of the theoretical period estimated using Equation (3).

Table 3. The swing period results of 10 runs of pendulum swinging.

\begin{tabular}{|c|c|c|c|}
\hline Run Name & $\begin{array}{l}\text { Period (s) Estimated Using the } \\
\text { Accelerometer's Readings }\end{array}$ & $\begin{array}{l}\text { Theoretical Period (s) } \\
\text { Estimated Using Equation (3) }\end{array}$ & $\%$ Error \\
\hline Run1 & 1.286 & \multirow{10}{*}{1.30} & 1.05 \\
\hline Run2 & 1.302 & & 0.15 \\
\hline Run3 & 1.265 & & 2.72 \\
\hline Run4 & 1.313 & & 1.02 \\
\hline Run5 & 1.312 & & 0.92 \\
\hline Run6 & 1.270 & & 2.31 \\
\hline Run7 & 1.298 & & 0.14 \\
\hline Run8 & 1.310 & & 0.77 \\
\hline Run9 & 1.285 & & 1.18 \\
\hline Run10 & 1.350 & & 3.85 \\
\hline
\end{tabular}

\subsection{Calibration on a Shaking Table}

The next experiment involves attaching the sensor to a shaking table with a back-and-forth movement of a known acceleration of $6 \mathrm{~g}$. The representative time series for the acceleration in the direction of movement (achieved by aligning the sensor's local coordinate system with that of the shake table) is shown in Figure 6.

The highest value recorded by the accelerometer is $6.27 \mathrm{~g}$. It is clear that any errors in this experiment are not due to the sensor alone but also because the sensor is not rigidly fixed on the shaking table, thus the full- and without-time delay and the transfer of motion cannot be practically achieved. The error for this experiment is $4.5 \%$, which is acceptable, considering that accelerations are typically far below this range for applications in water environments and for this size and submerged density of the instrumented particle. 


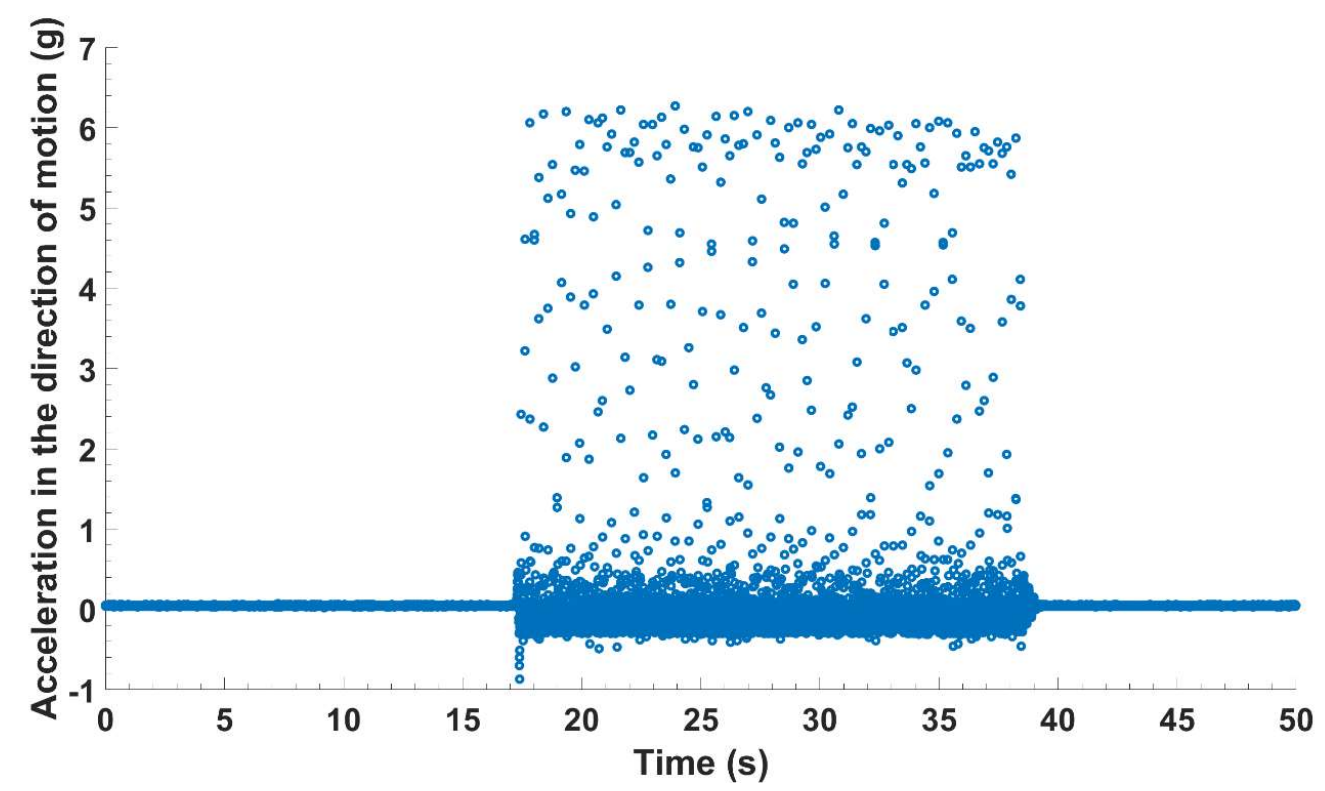

Figure 6. The accelerometer logged readings in the direction of motion of the shaking table.

\subsection{Calibration of Gyroscope for Pure Rolling along an Incline}

As for the gyroscope calibration, it consists of attaching the sensor to the centre of a hollow cylinder and letting it roll down an inclined plane of $5 \mathrm{~cm}$ in height, starting from rest, as illustrated in Figure 7.
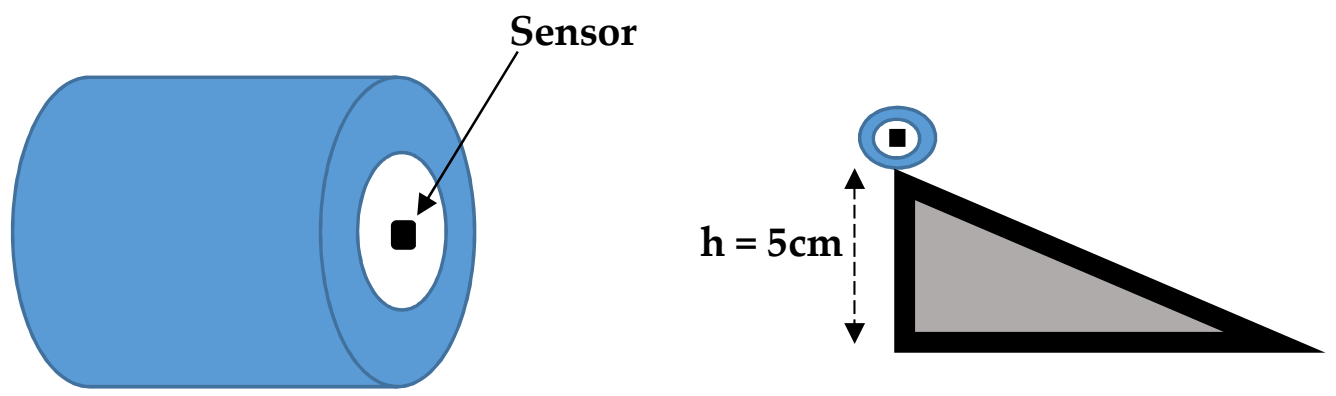

Figure 7. An illustration of rolling the sensor attached to the centre of a hollow cylinder down an inclined plane of $5 \mathrm{~cm}$ in height.

To ensure the repeatability of the experiment and minimise the experimental error, a thread is attached to the start location of the roll to ensure each run starts from the same position and a hard surface is placed at the end of the plane to keep the inclined plane in the same location. For a pure roll down an inclined plane, the conservation of energy can be used to estimate the angular velocity down the inclined plane since the potential lost by the drop in height should be equal to the gain in kinetic energy down the plane, as shown in Equation (4), assuming zero friction between the cylinder and plane surface:

$$
\mathrm{mgh}=\frac{1}{2} \mathrm{mv} v^{2}+\frac{1}{2} \mathrm{I} \omega^{2}
$$

where $\mathrm{m}$ is the mass, $\mathrm{h}$ is the height of the inclined plane, $\mathrm{v}$ is the linear velocity, $\mathrm{I}$ is the moment of inertia and $\omega$ is the angular velocity. The moment of inertia of the hollow cylinder depends on its inner and outer radii, as shown in Equation (5):

$$
\mathrm{I}=\frac{1}{2} \mathrm{~m}\left(\mathrm{a}^{2}+\mathrm{b}^{2}\right)
$$


where $a$ is the inner radius and $b$ is the outer radius of cylinder.

Substituting Equation (4) in Equation (5) and simplifying yields:

$$
\omega=\sqrt{\frac{2 g h}{b^{2}+\frac{1}{2}\left(b^{2}+a^{2}\right)}}
$$

with inner and outer radii of the hollow cylinder of 40 and $55 \mathrm{~mm}$, respectively, and a height of the inclined plane of $5 \mathrm{~cm}$, the angular velocity theoretically should be $13.55 \mathrm{rad} / \mathrm{s}$. The angular velocity is calculated using the sensor's gyroscope data as $\omega=\sqrt{\omega_{\mathrm{x}}^{2}+\omega_{\mathrm{y}}^{2}+\omega_{\mathrm{z}}^{2}}$. The maximum recorded angular velocity results by the gyroscope for the 10 runs are shown in Table 4 , which corresponds to the angular velocity at the bottom of the inclined plane, as shown in Figure 8. As for the sudden drop in the total angular velocity after it reached a maximum, this is due to the hard surface that is set at the bottom of the inclined plane.

Table 4. The total angular velocity results of 10 runs of pure rolling down an incline.

\begin{tabular}{lccc}
\hline Run Name & $\begin{array}{c}\text { Total Angular Velocity (rad/s) } \\
\text { Estimated Using the } \\
\text { Gyroscope's Readings }\end{array}$ & $\begin{array}{c}\text { Theoretical Total Angular } \\
\text { Velocity (rad/s) Estimated } \\
\text { Using Equation (6) }\end{array}$ & \% Error \\
\hline Run1 & 13.40 & & 1.11 \\
Run2 & 13.92 & & 2.73 \\
Run3 & 13.32 & & 1.70 \\
Run4 & 13.10 & 13.55 & 3.32 \\
Run5 & 13.65 & & 0.74 \\
Run6 & 13.42 & & 0.96 \\
Run7 & 14.30 & 5.54 \\
Run8 & 13.69 & & 1.03 \\
Run9 & 13.45 & 0.74 \\
Run10 & 13.25 & & 2.21 \\
\hline
\end{tabular}

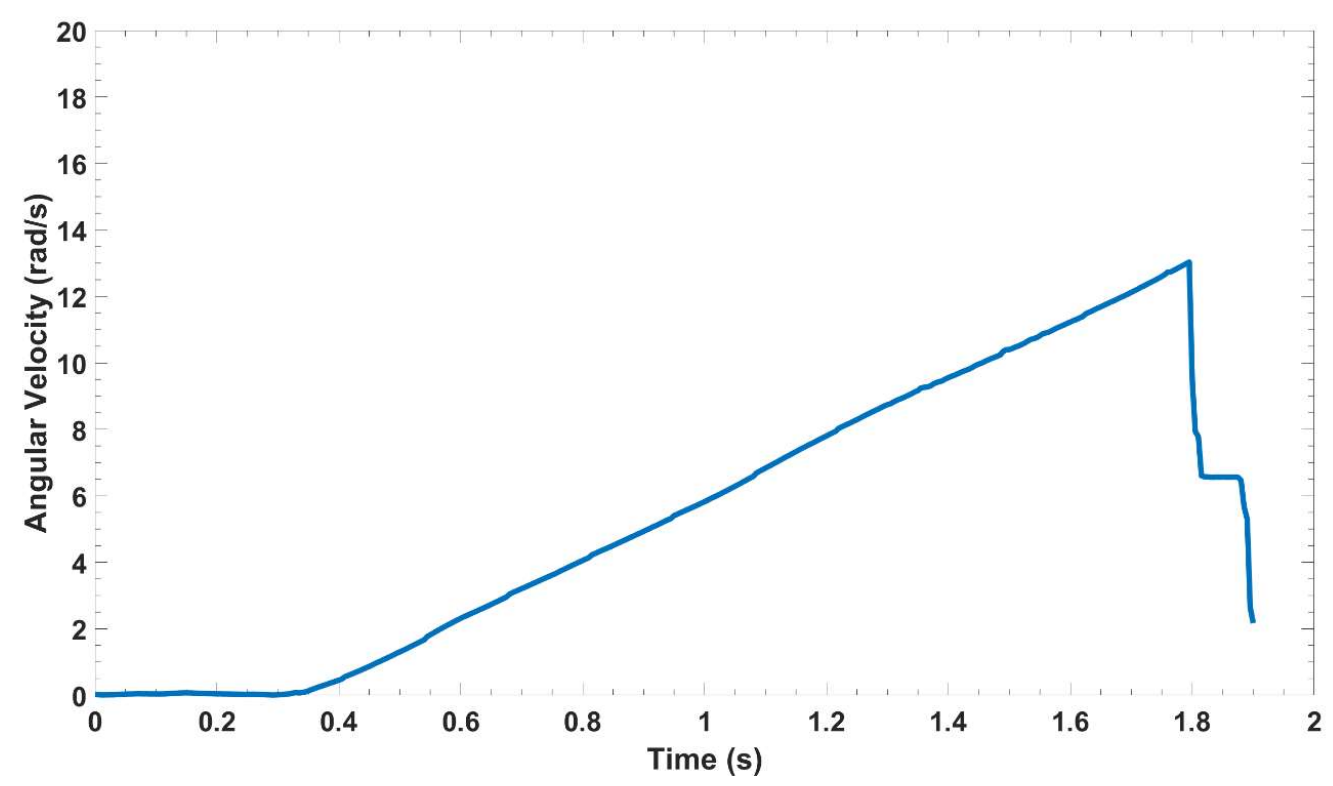

Figure 8. Total angular velocity recorded by the sensor during a roll from the top to the bottom of the inclined plane. 
The standard deviation for the 10 runs in the experiment is 0.335 , which is a small value, indicating a small spread in the results. The standard deviation per mean is estimated to be 0.0247 , indicating that the error in the experiment is small. The average error in the 10 runs is $2.01 \%$, which is deemed acceptable, considering the fact that the measurements are made by hand and the theoretical estimation using Equation (6) is based on the assumption that there is no sliding motion between the cylinder and the inclined plane. Additionally, the data used for the calculations are the raw data before the inertial sensor fusion, i.e., before using the accelerometer data to correct the gyroscope data since the gyroscope's data are less reliable due to drifting [26,27].

\subsection{Data Fusion}

The calibration of the accelerometer includes two different experiments: simple harmonic motion and using a shaking table, with average error values of $1.08 \%$ and $4.5 \%$, respectively. On the other hand, the calibration of the gyroscope includes pure rolls down an inclined plane experiment, with an error of $2.01 \%$. The range of errors arising from the sensor's readings and from the experimental measurements are quantified in this calibration process. The next step involves inverse uncertainty quantification by parameter calibration and data fusion. Specifically, this involves changing the parameters included in the input of a filter to be used for sensor data fusion to estimate the final corrected acceleration, angular velocity and orientation, based on the experimental results and the known information about the sensors. The filter itself uses nine-axis Kalman [28] filter structures with inputs of the expected accelerometer, gyroscope and magnetometer noise. It is decided to use the average of the mean square error of the pendulum period estimation and acceleration estimation of the pendulum swinging experiments as the accelerometer noise and the mean square error of pure rolls down an incline experiment as the gyroscope noise for the input of the inertial sensor fusion filter. Specifically, accelerometer noise of $0.0029608\left(\mathrm{~m} / \mathrm{s}^{2}\right)^{2}$ and gyroscope noise of $0.11238(\mathrm{rad} / \mathrm{s})^{2}$ are used as the inputs for the inertial sensor fusion filter.

\section{Testing}

\subsection{Flume Setup and Test Section}

In order to test the instrumented particle, a well-controlled laboratory experiment is performed in an $8 \mathrm{~m}$ long water recirculating flume. This $0.9 \mathrm{~m}$ wide water recirculating open channel is able to carry flows of up to $0.4 \mathrm{~m}$ deep, while water is provided with a maximum capacity of $0.2 \mathrm{~m}^{3} / \mathrm{s}$, controlled by a torque inverter though which the operating frequency of the pump can be adjusted. In order to artificially create the intended flow depths at reasonable flow velocities, an adjustable tailgate is located at the outlet, while the bed surface slope can be adjusted. In order to achieve an adequate hydraulic roughness, the bed surface is paved with a few layers of water-worked uniformly sized fine gravel with a median size of about $\mathrm{d}_{50}=15-25 \mathrm{~mm}$.

The test section (has a length of $1 \mathrm{~m}$ ) is located in the downstream section of the flume, approximately $5.25 \mathrm{~m}$ downstream from the inlet and $1.75 \mathrm{~m}$ upstream from the outlet (Figure 9a), in order to ensure that the hydraulically rough turbulent flow is fully developed. The test section is positioned along the centreline of the flume, $45 \mathrm{~cm}$ from either of the flume's side glass walls. An illustration of the flume with the instrumented particle and local bed microtopography is shown in Figure 9b, demonstrating the instrumented particle's initial and displaced position. 


\section{Flume}

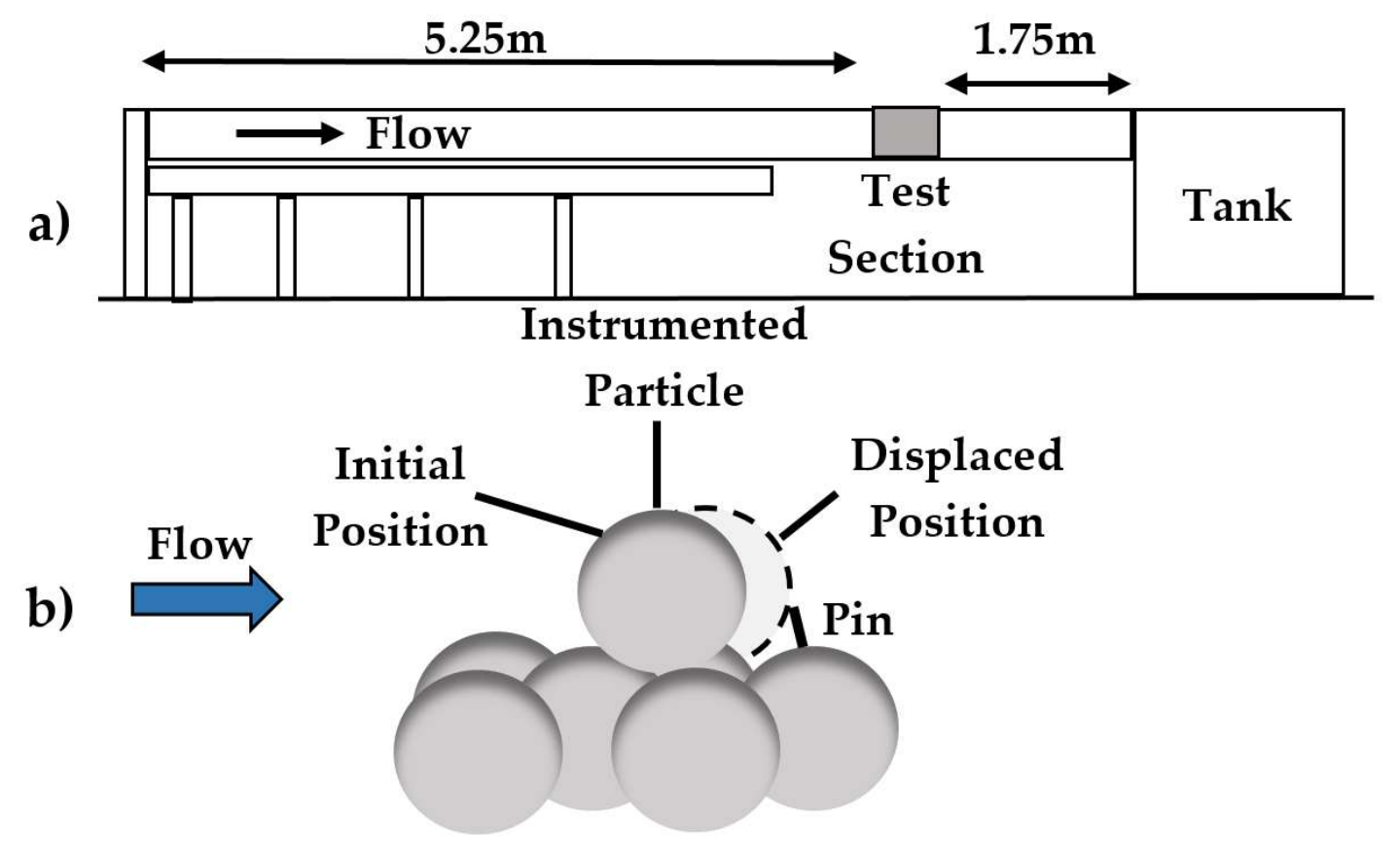

Figure 9. Schematic of the (a) experimental set-up showing the relative location of the test section and $(\mathbf{b})$ test section, showing the instrumented particle resting on top of the tetrahedral bed particle arrangement (side view).

\subsection{Local Microtopography}

A 3D-printed bed micro-topography, consisting of four hemispheres in a rectilinear well-packed arrangement, is designed to position the spherical instrumented particle of $4 \mathrm{~cm}$ in diameter to study its turbulent flow induced entrainments. The particle is sitting on the local micro-topography, which is embedded within the bed surface, and is allowed to move from the upstream towards the downstream pocket due to sufficiently energetic instantaneous near bed surface flow structures. However, full entrainment is restrained due to a pin $2 \mathrm{~mm}$ downstream of the instrumented particle. With this method, the particle falls back to its resting location after the advection of energetic flow events pass it, thus enabling a continuous series of entrainments without disturbing the experiment by manually moving the particle back to its initial position $[5,7]$.

\subsection{The Instrumented Particle}

The sensor's inertial measurement unit (IMU) is located at the centre of a $4 \mathrm{~cm}$ diameter spherical enclosure that maintains the electronic components waterproofed during testing, and ensures they are not subjected to excessive stresses. A range of different particle shapes and sizes of enclosures can be used depending on the objective. Having the sensor at the centre of mass, surrounded by equally distributed weights of lead, a uniform distribution of mass is obtained, so that the instrumented particle's motion is not biased and any offsets in the readings are avoided.

The casing is designed to be versatile to accommodate different amount of weights. It is important to have an adjustable density for different flow regimes, as the resistance to flow-induced entrainment is directly proportional to the particle's submerged weight. A density of $2.65 \mathrm{~g} / \mathrm{cm}^{3}$ is used for the testing, which is the density of the quartz earth sediment typically found in nature. The casing designs are produced using SolidWorks ${ }^{\mathrm{TM}}$ and are built using a rigid opaque photopolymer material and additive manufacturing (3D-printing) equipment (Formlabs, Somerville, MA, USA). 


\subsection{Experimental Protocol}

A high-speed video camera, operating at 120 frames per second, is placed on one side of the flume near the test section in order to record the particle's movements accurately. The waterproof camera is of minimum dimensions $(2 \times 2 \mathrm{~cm})$ and placed slightly downstream of the test section in order to avoid any interference with the flow properties near the test section that could affect the instrumented particle's response. An ultra-bright light source is placed above the test section, and a high contrast black material is placed in the background of the camera's viewing area in order to enable accurate monitoring.

The flume is run at a flowrate that results in a continuous entrainment of the instrumented particle, which is confirmed by eye, i.e., the particle remained entrained and is stopped from moving further by the pin that is set in place. Then, the flowrate is reduced incrementally to finally have three flowrates that result in some periodic entrainments. A Nortek Vectrino1 acoustic Doppler velocimetry (ADV) is used to obtain the velocity profiles $10 \mathrm{~cm}$ upstream of the test section. The total time of the experiment is about $1 \mathrm{~h}$, with the flow measurements being made after removing the test section to prevent any interference. Each of the three runs involves $10 \mathrm{~min}$ flow measurement recordings and $10 \mathrm{~min}$ sensor recordings. The sensor logs for about an hour at a frequency of $200 \mathrm{~Hz}$, which serves as another confirmation that the instrumented particle can be deployed to record data for a sufficiently long time.

\section{Results and Discussion}

A proper filter, with the input of accelerometer noise and gyroscope noise values changing based on the calibration results, as discussed in Section 3.5, is used for the inertial sensor fusion. The results of sensor fusion are then used to calculate the corrected total acceleration for the three runs using Equation (1). For the three runs, the corrected total acceleration results are investigated and compared to the video recordings of the side camera. Based on a manual comparison between the sensor's data and the video recordings, a threshold value for the total acceleration is obtained by ensuring that the peaks in the total acceleration, corresponding to full entrainment events and twitching, are above and below this line, respectively. An illustration of how the corrected total acceleration results could be used to detect the range of the instrumented particle's frequencies is shown in Figure 10 below.

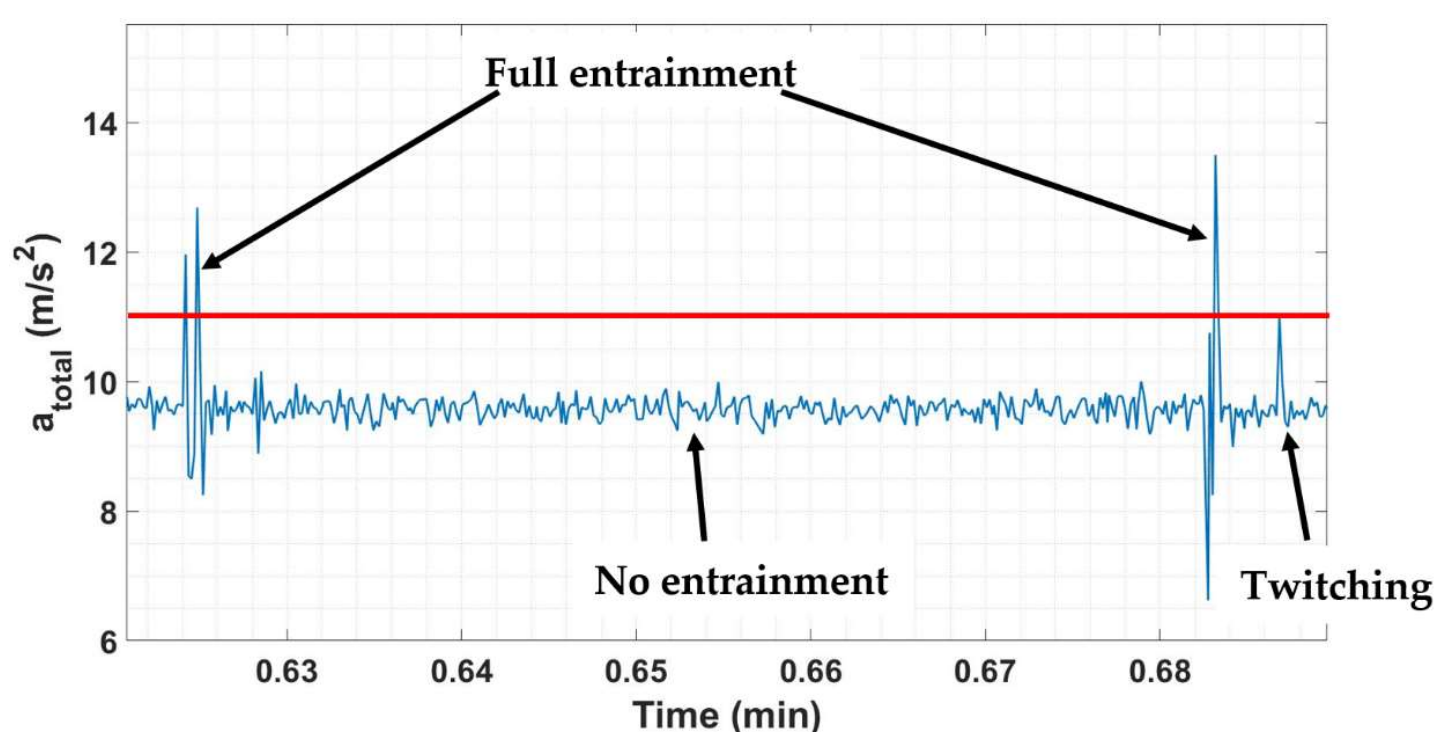

Figure 10. An illustration of how the fused sensor readings (corrected total acceleration results specifically) could be used to detect the range of the instrumented particle's frequencies. The thick red line represents a threshold-total acceleration value. 
In Figure 10, a total acceleration value of almost $9.81 \mathrm{~m} / \mathrm{s}^{2}$ indicates no entrainment of the instrumented particle or that no energetic event acts on the instrumented particle by turbulent flowing water that is strong enough to overcome its resistance. In other words, the particle is at its resting position and the accelerometer records the gravitational acceleration in the z-direction and records zero in the $\mathrm{x}$ - and y-directions. At $0.688 \mathrm{~min}$, the total acceleration results show a relatively small spike, indicating that there is a small energetic event(s) that could not result in the full entrainment of the instrumented particle, but rather in twitching it only. On the other hand, the total acceleration values are greater than exceedance (or the threshold-total acceleration value) at $0.625 \mathrm{~min}$ and $0.683 \mathrm{~min}$, which indicate full entrainment events of the instrumented particle. At each instance of both events, there is an energetic event(s) that is strong enough to dislodge the instrumented particle from its resting position and move it against the restraining pin. The procedure, illustrated in Figure 10, is applied to the total acceleration results of the three runs by eliminating the values that are below a threshold-total acceleration value of $11 \mathrm{~m} / \mathrm{s}^{2}$ from the analysis. In other words, only full entrainment events are left to derive the frequency of entrainment of the instrumented particle for the three runs of the experiment, as shown in Figure 11 below.

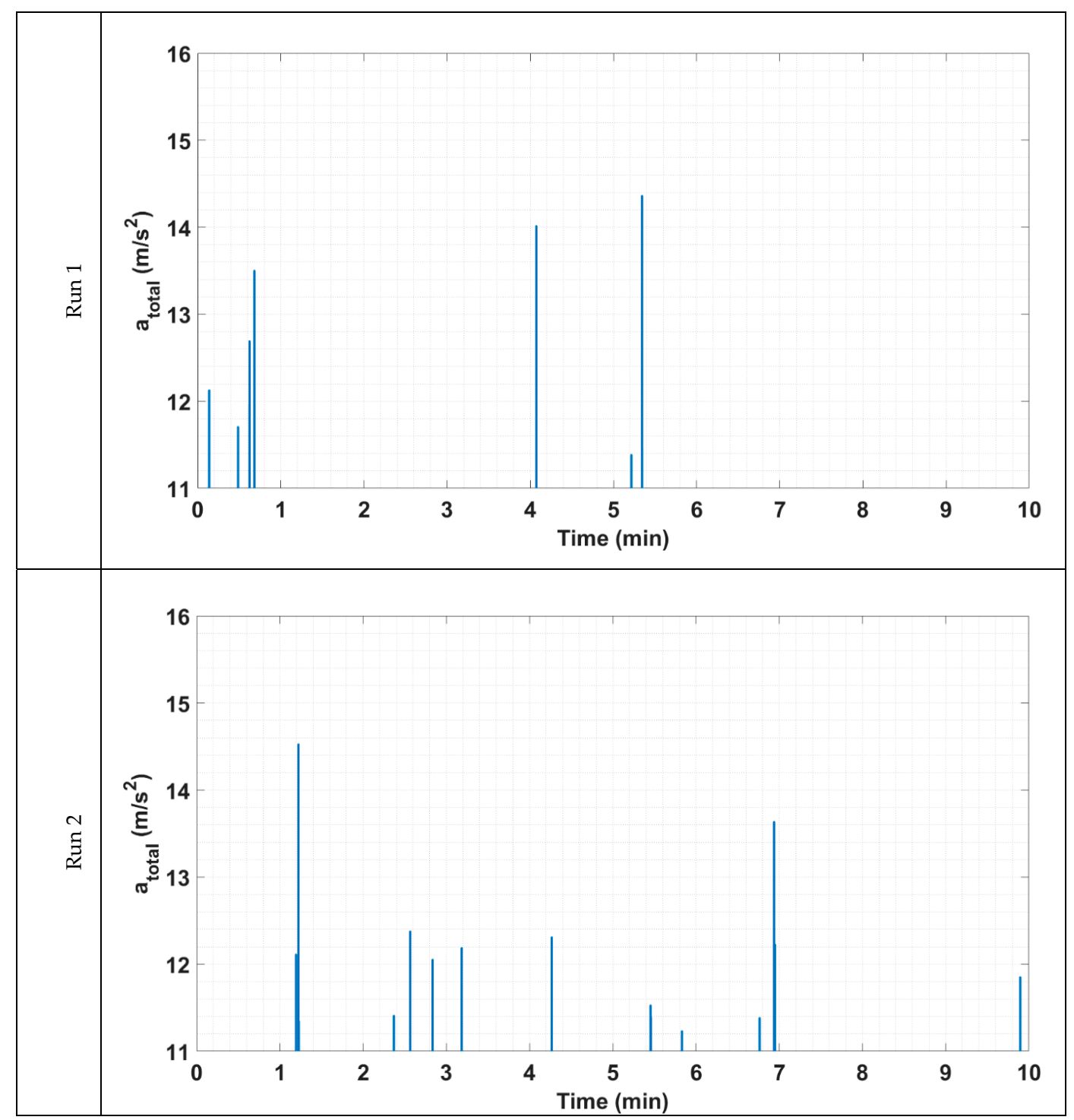

Figure 11. Cont. 


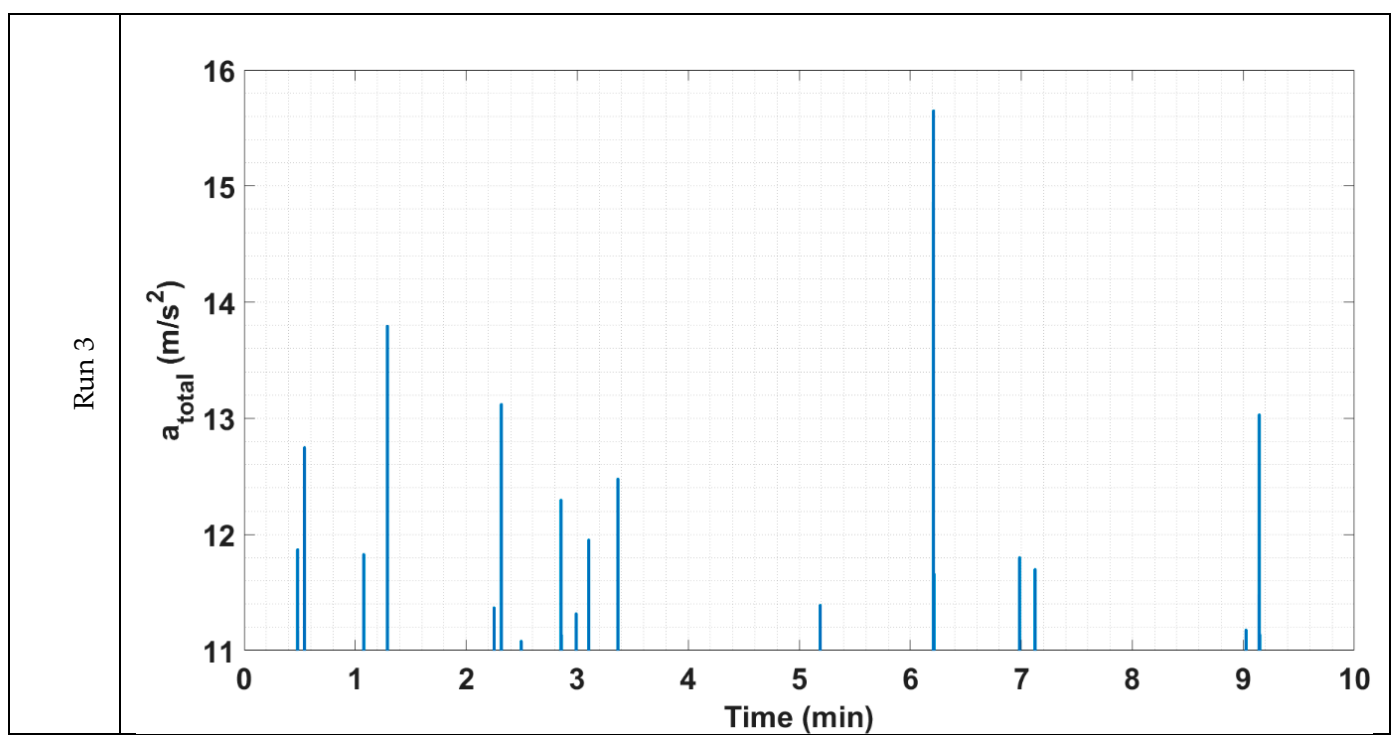

Figure 11. Above threshold-corrected total acceleration results for the three runs of the experiment.

Using the results shown in Figure 11, the frequency of entrainment, $\mathrm{f}_{\mathrm{E}}$, of the instrumented particle for each run is calculated by dividing the total number of full entrainments by the total run time in seconds. These calculations were performed, and the results are shown in Table 5. The results are consistent with the experimental findings of Valyrakis et al. [6] for the frequency of entrainment of a coarse particle for near-threshold conditions. As for the flow velocity measurements, they are used to estimate the shear or friction velocity $\left(u_{*}\right)$ as shown in Table 5 using the Townsend method [29], which is based on the law of wall. The shear velocity, water properties, and particle diameter are used to estimate boundary, or particle, Reynolds number $\left(\operatorname{Re}_{*}\right)$ for each run as shown in Table 5 . The shear velocity and water density are then used to calculate the bed shear stress $\left(\tau_{0}\right)$ exerted by flowing water, which is used in addition to the particle's submerged weight $\left(\mathrm{W}_{\mathrm{s}}\right)$ to estimate Shields number or Shields parameter [30]. Shields parameter $\left(\tau_{*}\right)$ is a dimensionless number that is basically the ratio of fluid force exerted on the particle to the apparent or submerged weight of the particle. It is the most used criterion in the literature to determine whether there is initiation of motion of a sediment particle. Based on the results shown in Table 5, Shields parameter values for the three runs of the experiment are around the regime of incipient flow conditions predicted by a plethora of experiment studies (see [31,32], amongst others). Additionally, Nikuradse's equivalent sand roughness, which is an indicative parameter of the friction matched against an equivalent sand-grain roughness of height $k_{s}$, is estimated for the three runs of the experiment and results are presented in Table 5.

Table 5. Description and results of the three runs of the experiment.

\begin{tabular}{ccccccc}
\hline Run Name & $\mathbf{R} \mathbf{e} *=\frac{\boldsymbol{\rho u} * \mathbf{D}}{\mu}$ & $\mathbf{u} *(\mathbf{m m} / \mathbf{s})$ & $\mathbf{k}_{\mathbf{s}}(\mathbf{m m})$ & $\boldsymbol{\tau}_{\mathbf{o}}=\boldsymbol{\rho} \mathbf{u}_{*}^{2}(\mathbf{P a})$ & $\boldsymbol{\tau}_{*}=\frac{\boldsymbol{\tau}_{\mathbf{o}}}{\left(\boldsymbol{\rho}_{\mathbf{s}}-\boldsymbol{\rho}_{\mathbf{w}}\right) \mathbf{g D}}$ & $\mathbf{f}_{\mathrm{E}}(\mathbf{H z})$ \\
\hline Run1 & 1810.3 & 0.040 & 13.65 & 1.63 & 0.00252 & 0.017 \\
Run2 & 1943.5 & 0.043 & 18.47 & 1.88 & 0.00290 & 0.028 \\
Run3 & 2024.2 & 0.045 & 20.73 & 2.03 & 0.00313 & 0.042 \\
\hline
\end{tabular}

It is clear from the results shown in Figure 11 and Table 5 that the frequency of entrainment of the instrumented particle, $\mathrm{f}_{\mathrm{E}}$, increases with increasing the flowrate (or boundary Reynold's number). Additionally, it is clear from the results presented in Table 5 that frequency of entrainment of the instrumented particle, $\mathrm{f}_{\mathrm{E}}$, increases with increasing mean Shields stresses. Based on Figures 10 and 11 and Table 5, the logged readings by the sensors could be fused to detect different types of motion of the particle accurately without the need for visual observations. Specifically, full entrainment, twitching, and no entrainment could be detected using the total acceleration results after defining a 
threshold value. Additionally, the corrected total acceleration results could be used to estimate the entrainment frequency of the instrumented particle. Such a metric could be linked to the probability of entrainment of individual sediment particles. Therefore, the instrumented particle provides a direct and cost-effective tool for monitoring the initiation of transport processes of coarse sediment particles in turbulent environments. This is of practical relevance as visual observation methods are often restricted in turbulent environments (and commonly high turbidity environments).

There are a number of tools developed to monitor the actual occurrence of scour around hydraulic infrastructure [33-35]. However, many geomorphological and hydrological hazards, such as scour around hydraulic infrastructure or riverbed and riverbank destabilisation, typically develop very fast relative to our capacity to take action once they are initiated or detected. Therefore, assessing the probability of the occurrence of the start of scour processes, before the conditions for any further critical catastrophes or infrastructure failure start setting in, is of interest.

While tools developed in the literature are designed to assess scour in real time, the tool offered herein is designed following a different approach: monitoring the risk of surface particle removal. This inherently links to well-established theories in hydraulic engineering around the probability of entrainment of individual sediment particles (see [6], amongst others). According to such probabilistic approaches, monitoring the frequency of entrainments of the most-exposed particle resting on the bed surface in the vicinity of the hydraulic infrastructure of interest should suffice to detect the risk of the onset of scour occurring before it actually takes place. In other words, monitoring the probability of particle destabilisation (or probability of entrainment) from the exceedances of acceleration, or instances where acceleration is greater than exceedance. Thus, this research focuses on the development, calibration and demonstration of the utility of the instrumented particle for assessing the probability of the onset of scour. The results from the flume experiments presented herein showcase that, by placing the instrumented particle at an appropriate location at a distance scaling with the dimension of the infrastructure (e.g., bridge pier diameter) downstream of it, offers a tool to assess the risk of scour initiation.

Additionally, performance indicators relevant to bed stability or the risk for scour can be derived from the demonstrated sensor fusion of the recordings of the inertial sensor, using, for example, metrics such as the rate of entrainment or the frequency of entrainment for the instrumented particle. This can be derived by simply estimating the number of full entrainments over the total logging period and serves as a probabilistic estimation of the risk of the destabilisation of bed material or the potential of scour development around built infrastructure. When combining such results with machine learning and algorithms, hybrid advanced data-driven predictive models can be developed in the future to assess water infrastructure risk and determine the potential for bed surface destabilisation $[5,6]$. Therefore, the instrumented particle has the potential to become a vital addition in the arsenal of sensors used for structural health monitoring, providing an early (well before any critical failures may appear), robust, rapid and cost-effective assessment of risks to the structural integrity of the infrastructure near water.

Furthermore, the results of the inertial sensor fusion of the logged readings could be used for estimating the near bed surface instantaneous hydrodynamic forces. Thus, it could help researchers to obtain a better understanding of the fundamental transport processes relevant to a plethora of fields, ranging from ecohydraulics to geomorphology. Additionally, experimentation with instrumented particles, as shown herein, can produce a large range of datasets, which can be used to benefit the numerical modelling of solids transport processes via validating Lagrangian particle transport models, including discrete element modelling (DEM), and can also be coupled with computational fluid dynamics (CFD-DEM) studies.

\section{Conclusions}

Electronic system integration at this micro-scale and with this complexity is a difficult task, however, it is greatly rewarding, as the developed instrumented particle is quite versatile and has 
a considerable and promising potential for future applications in the field of ecohydraulics and geosciences, as well as for all earth surface hazard sciences pursuing lab, field or numerical modelling techniques that can benefit from validating their Lagrangian particle transport models.

The newly produced instrumented particle design features a significantly reduced size $(40 \mathrm{~mm}$ compared to past versions of instrumented particles), proving that it is entirely possible to create a miniaturised user-friendly device (instrumented particle) that allows for monitoring bed surface destabilisation or local scour in a non-intrusive and direct manner.

The calibration process for the sensors is performed with quantifying the uncertainties in the process and the sensor's readings and the results of the calibration are used to determine the input for the inertial sensor fusion filter. The sensor is also tested in a recirculating open channel with a controlled water flow, flume and the results of the fusion of the testing experiments are shown and discussed. Such a low-cost device can have a wide range of applications in water engineering and infrastructure monitoring, especially in harsh and remote environments, allowing for a better capacity for the extraction of performance indicators (such as the intensity or frequency of particle motion). The rate of entrainment as a performance indicator can, if combined with machine learning and artificial intelligence algorithms, be used to provide predictive models that can be used to statistically determine the risk of destabilisation of the bed surface.

Future work includes considering more versatile ways for transferring data using frameworks such as the Internet of Things (IoT), where data can be locally transmitted to the base where the instrumented particle rests, then out of the water body via waterproof cables and via satellite (3GPRS) and delivered to the centre of monitoring operations. Additionally, algorithms to provide real-time analysis of data, which could be of a great value, especially for assessing the risk of scour in remote and harsh environments, are to be considered. Finally, even further miniaturisation to widen the applications of the instrumented particle is currently under consideration.

Supplementary Materials: The following are available online at https://susy.mdpi.com/user/submission/video/ d6a75069d08308f481caa1fccb6a803e, Video S1: Video Abstract.

Author Contributions: Conceptualization, M.V.; Data curation, K.A.-O. and Y.X.; Formal analysis, K.A.-O.; Funding acquisition, M.V.; Investigation, K.A.-O., Y.X. and M.V.; Methodology, K.A.-O. and M.V.; Project administration, M.V.; Software, K.A.-O.; Supervision, M.V.; Validation, K.A.-O.; Visualization, K.A.-O. and M.V.; Writing_-original draft, K.A.-O. and M.V.; Writing—review and editing, K.A.-O., Y.X. and M.V. All authors have read and agreed to the published version of the manuscript.

Funding: This research was supported by the Royal Society (Research Grant RG2015 R1 68793/1) and the Royal Society of Edinburgh (Crucible Award).

Conflicts of Interest: The authors declare no conflict of interest.

\section{References}

1. Valyrakis, M.; Alexakis, A.; Pavlovskis, E. "Smart pebble" designs for sediment transport monitoring. In Proceedings of the 17th EGU General Assembly (EGU2015), Vienna, Austria, 12-17 April 2015.

2. Valyrakis, M.; Alexakis, A. Development of a "smart-pebble" for tracking sediment transport. In Proceedings of the International Conference on Fluvial Hydraulics (River Flow 2016), St. Louis, MO, USA, 12-15 July 2016.

3. Valyrakis, M.; Farhadi, H. Investigating coarse sediment particles transport using PTV and "smart-pebbles" instrumented with inertial sensors. In Proceedings of the 19th EGU General Assembly (EGU2017), Vienna, Austria, 23-28 April 2017.

4. Izadinia, E.; Heidarpour, M.; Schleiss, A. Investigation of turbulence flow and sediment entrainment around a bridge pier. Stoch. Environ. Res. Risk Assess. 2012, 27, 1303-1314. [CrossRef]

5. Valyrakis, M.; Diplas, P.; Dancey, C.L.; Greer, K.; Celik, A.O. Role of instantaneous force magnitude and duration on particle entrainment. J. Geophys. Res. Earth Surf. 2010, 115, 1-18. [CrossRef]

6. Valyrakis, M.; Diplas, P.; Dancey, C.L. Entrainment of coarse grains in turbulent flows: An extreme value theory approach. Water Resour. Res. 2011, 47, 1-17. [CrossRef]

7. Valyrakis, M.; Diplas, P.; Dancey, C.L. Entrainment of coarse particles in turbulent flows: An energy approach. J. Geophys. Res. Earth Surf. 2013, 118, 42-53. [CrossRef] 
8. Liu, D.; Valyrakis, M.; Williams, R. Flow Hydrodynamics across Open Channel Flows with Riparian Zones: Implications for Riverbank Stability. Water 2017, 99, 720. [CrossRef]

9. Diplas, P.; Celik, A.O.; Valyrakis, M.; Dancey, C.L. Some Thoughts on Measurements of Marginal Bedload Transport Rates Based on Experience from Laboratory Flume Experiments. In U.S. Geological Survey Scientific Investigations Report 2010-5091; United States Geological Survey: Reston, VA, USA, 2010; pp. 130-142.

10. Koursari, E.; Wallace, S.; Valyrakis, M.; Michalis, P. Remote Monitoring of Infrastructure at Risk due to Hydrologic Hazards and Scour. In Proceedings of the 21st EGU General Assembly (EGU2019), Vienna, Austria, 7-12 April 2019.

11. Papanicolaou, T.; Knapp, D. A particle tracking technique for bedload motion. In Proceedings of the 7th International Conference on HydroScience and Engineering (ICHE2006), Philadelphia, PA, USA, 10-13 September 2006.

12. Akeila, E.; Salcic, Z.; Kularatna, N.; Melville, B.; Dwivedi, A. Testing and calibration of smart pebble for river bed sediment transport monitoring. In Proceedings of the IEEE Sensors, Atlanta Georgia, GA, USA, 28-31 October 2007.

13. Akeila, E.; Salcic, Z. Smart Pebble for Monitoring Riverbed Sediment Transport. IEEE Sens. J. 2010, 10, 1705-1717. [CrossRef]

14. Kularatnal, N.; Kularatna-Abeywardana, D. Use of motion sensors for autonomous monitoring of hydraulic environments. In Proceedings of IEEE Sensors, Lecce, Italy, 26-29 October 2008.

15. Gronz, O.; Hiller, P.; Wirtz, S.; Becker, K.; Iserloh, T.; Seeger, M.; Brings, C.; Aberle, J.; Casper, M.; Ries, J. Smartstones: A small 9-axis sensor implanted in stones to track their movements. Catena 2016, 142, 245-251. [CrossRef]

16. Frank, D.; Foster, D.; Sou, I.; Calantoni, J.; Chou, P. Lagrangian measurements of incipient motion in oscillatory flows. J. Geophys. Res. Oceans 2014, 120, 244-256. [CrossRef]

17. Zhang, Y.; Ghannam, R.; Valyrakis, M.; Heidari, H. Design and Implementation of a 3D Printed Sensory Ball for Wireless Water Flow Monitoring. In Proceedings of the 4th International Conference on UK-China Emerging Technologies, Glasgow, UK, 21-22 August 2019; pp. 1-4.

18. Maniatis, G.; Hoey, T.; Hassan, M.; Sventek, J.; Hodge, R.; Drysdale, T.; Valyrakis, M. Calculating the Explicit Probability of Entrainment Based on Inertial Acceleration Measurements, J. Hydraul. Eng. 2017, 143, 1-43. [CrossRef]

19. Noss, C.; Koca, K.; Zinke, P.; Henry, P.H.; Navaratnam, C.U.; Aberle, J.; Lorke, A. A Lagrangian drifter for surveys of water surface roughness in streams. J. Hydraul. Res. 2019, 1-18. [CrossRef]

20. Houston, C.; Muir, D.; Valyrakis, M. Infrastructure scour risk assessment using instrumented particles. In Proceedings of the UK/China Emerging Technologies (UCET), Glasgow, UK, 21-22 August 2019.

21. MPU-9250 Product Specification Revision 1.1. Available online: https:/www.invensense.com/wp-content/ uploads/2015/02/PS-MPU-9250A-01-v1.1.pdf (accessed on 21 November 2019).

22. CoinPower CP 1654 A3 Datasheet. Available online: https://products.varta-microbattery.com/applications/ mb_data/documents/data_sheets/DS63165_3.pdf (accessed on 30 January 2018).

23. DTP301120 Product Specification. Available online: http://cdn.sparkfun.com/datasheets/Prototyping/SPE-00301120-40mah-en-1.0ver.pdf (accessed on 28 January 2018).

24. Rechargable Lithium-ion Cell. Available online: https:/products.varta-microbattery.com/en/products/ batteries-cells-configurations/technology/rechargeable/lithium-button-cells/all/technology-description. html (accessed on 29 January 2018).

25. Pendrill, A.; Rohlén, J. Acceleration and rotation in a pendulum ride, measured using an iPhone 4. Phys. Educ. 2011, 46, 676-681. [CrossRef]

26. Cechowicz, R. Bias Drift Estimation for MEMS Gyroscope Used in Inertial Navigation. Acta Mech. Autom. 2017, 11, 104-110. [CrossRef]

27. Xing, H.; Hou, B.; Lin, Z.; Guo, M. Modeling and Compensation of Random Drift of MEMS Gyroscopes Based on Least Squares Support Vector Machine Optimized by Chaotic Particle Swarm Optimization. Sensors 2017, 17, 2335. [CrossRef] [PubMed]

28. Kalman, R.E. A New Approach to Linear Filtering and Prediction Problems. J. Basic Eng. 1960, 82, 35-45. [CrossRef]

29. Towsend, A.A. The Structure of Turbulent Shear Flow, 2nd ed.; The Press Syndicate of the University of Cambridge: Cambridge, UK, 1980. 
30. Shields, A. Application of Similarity Principles and Turbulence Research to Bed-Load Movement; California Institute of Technology: Pasadena, CA, USA, 1936.

31. Buffington, J.; Montgomery, D. A systematic analysis of eight decades of incipient motion studies, with special reference to gravel-bedded rivers. Water Resour. Res. 1997, 33, 1993-2029. [CrossRef]

32. Pähtz, T.; Clark, A. H.; Valyrakis, M.; Durán, O. The Physics of Sediment Transport Initiation, Cessation, and Entrainment Across Aeolian and Fluvial Environments. Rev. Geophys. 2020, 58, 1-58. [CrossRef]

33. Michalis, P.; Tarantino, A.; Tachtatzis, C.; Judd, D.M. Wireless Monitoring of Scour and Re-deposited Sediment Evolution at Bridge Foundations based on Soil Electromagnetic Properties. Smart Mater. Struct. 2015, 24, 1-15. [CrossRef]

34. Chen, G.; Schafer, B.P.; Lin, Z.; Huang, Y.; Suaznabar, O.; Shen, J.; Kerenyi, K. Maximum scour depth based on magnetic field change in smart rocks for foundation stability evaluation of bridges. Struct. Health Monit. 2015, 14, 86-99. [CrossRef]

35. Tang, F.; Chen, Y.; Guo, C.; Fan, L.; Chen, G.; Tang, Y. Field Application of Magnet-based Smart Rock for Bridge Scour Monitoring. J. Bridg. Eng. 2019, 24. [CrossRef]

C 2020 by the authors. Licensee MDPI, Basel, Switzerland. This article is an open access article distributed under the terms and conditions of the Creative Commons Attribution (CC BY) license (http://creativecommons.org/licenses/by/4.0/). 